\title{
Noninvasive evaluation of internal thoracic artery and left anterior descending coronary artery anastomotic sites using transthoracic Doppler echocardiography: Comparison with coronary arteriography
}

Nobuaki Hirata, MD

Nobuaki Asaoka, PhD

Akira Amemiya, MD

Shinichi Hatsuoka, MD

Takayoshi Ueno, MD

Yoshio Kosakai, MD

\footnotetext{
From the Division of Cardiovascular Surgery, Takarazuka Municipal Hospital, Takarazuka, Hyogo, Japan.

Received for publication Feb 19, 2002; revisions requested July 8, 2002; revisions received Nov 8, 2002; accepted for publication Dec 4, 2002.

Address for reprints: Nobuaki Hirata, MD, Division of Cardiovascular Surgery, Takarazuka Municipal Hospital, 4-5-1, Kohama, Takarazuka, Hyogo 665-0827, Japan (E-mail: hirata@xd5.so-net.ne.jp).

J Thorac Cardiovasc Surg 2003;126:1080-6

Copyright $(2) 2003$ by The American Association for Thoracic Surgery

$0022-5223 / 2003 \$ 30.00+0$

doi:10.1016/S0022-5223(03)00812-2
}

Objectives: This study was designed to evaluate anastomotic sites located between the internal thoracic artery and left anterior descending coronary artery using transthoracic Doppler echocardiography, and then to clarify the accuracy of those results by comparison with coronary arteriographic findings.

Methods: We examined 35 consecutive patients who had undergone bypass surgery. The echocardiographic examinations were performed within approximately 1 week of follow-up coronary arteriography, which occurred at $4.3 \pm 2.2$ months after bypass surgery. We measured the diameter using intraluminal flow signals, and we also measured flow velocity.

Results: Adequate spectral Doppler recordings of coronary flow in the anastomosis were obtained in 31 (89\%) of the 35 study patients. In the normal anastomosis group $(n=25)$, the diameter and the peak blood flow velocity of the internal thoracic artery and left anterior descending coronary artery were $1.5 \pm 0.3 \mathrm{~mm}$ and $2.0 \pm$ $0.4 \mathrm{~mm}$, and $58 \pm 25 \mathrm{~cm} / \mathrm{s}$ and $47 \pm 20 \mathrm{~cm} / \mathrm{s}$, respectively. Stringed internal thoracic artery was found in 4 patients; the echocardiographic findings revealed a greater amount of information regarding the physiologic state in the area of anastomosis compared with angiographic findings. In a stenotic anastomosis found in 2 patients, the blood flow velocity findings at the anastomotic sites $(83 \pm 228$ $\mathrm{cm} / \mathrm{s})$ were higher than those in normal anastomotic patients $(59 \pm 28 \mathrm{~cm} / \mathrm{s})$.

Conclusions: Transthoracic Doppler echocardiography enabled an effective evaluation of anastomotic sites between the internal thoracic artery and left anterior descending coronary artery in over $80 \%$ of our patients. This totally noninvasive method is thought to be reliable and able to provide a greater amount of information, compared with coronary arteriography, regarding the physiologic state of an anastomosis, such as a competitive relationship.

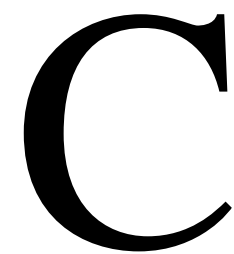
oronary artery bypass graft (CABG) surgery is a widely used therapeutic procedure for symptomatic coronary artery disease. The internal thoracic artery (ITA) is now considered the graft of choice because of its high rate of long-term patency. ${ }^{1,2}$ Use of at least one ITA in an anastomosis to the left anterior descending coronary artery (LAD) has been shown to result in a reduction in risk of late cardiac events and an improvement of survival. ${ }^{3}$ Currently, the patency of a CABG is usually assessed by coronary arteriography. 
In a previous study, approaches from the subclavian fossa and/or intercostal spaces using duplex Doppler echocardiography were found to be feasible for noninvasive evaluations of left ITA graft blood flow. ${ }^{4,5}$ However, those methods were limited to examination of the flow at the stem of the ITA; we consider the most important point of an ITA-LAD anastomosis to be the anastomotic site.

Several studies have reported that epicardial coronary blood flow velocity can be measured by transthoracic Doppler echocardiography, which is noninvasive and widely used in clinical settings. ${ }^{6-8}$ Recent technological advancements in color Doppler echocardiography may provide a high success rate when evaluating anastomotic sites located between the ITA and LAD by measuring coronary flow velocity.

The purpose of this study was to evaluate anastomoses located between the ITA and LAD using transthoracic Doppler echocardiography, and then to clarify the accuracy of the results by comparing them with those using coronary arteriography.

\section{Patients and Methods Study Patients}

We examined 35 consecutive patients who had undergone CABG using the ITA to LAD and who received a postoperative quantitative coronary arteriographic examination during the period from July 1999 to April 2001. The patients consisted of 27 men and 8 women, with a mean age of $64 \pm 12$ years old (ranging from 38 to 78 years), whose body weight and body surface area were 59.9 $\pm 9.8 \mathrm{~kg}$ (ranging from 40 to $75.5 \mathrm{~kg}$ ) and $1.60 \pm 0.15 \mathrm{~m}^{2}$ (ranging from 1.30 to $1.80 \mathrm{~m}^{2}$ ), respectively.

Twenty-eight patients underwent CABG because of angina pectoris and 7 because of post-myocardial infarction angina. Before bypass grafting, the viability of the anterior wall was confirmed in all patients by stress radionuclide imaging. Bypass grafting was performed at the midportion of the LAD in all patients. In the present study, 29 patients underwent CABG using a traditional cardiopulmonary bypass, and 6 patients underwent CABG without cardiopulmonary bypass (off-pump CABG). The number of grafts per patients in this series was $3.8 \pm 1.5$. The time interval between bypass grafting and coronary arteriography was $4.3 \pm 2.2$ months (mean 1.0 to 9.0 months). There were no cases of atrial fibrillation or previous CABG.

All participants were approved by the Committee for the Protection of Human Subjects in Research of Takarazuka Municipal Hospital.

\section{Transthoracic Doppler Echocardiographic Studies}

The echocardiographic procedures were performed within approximately 1 week of postoperative follow-up coronary arteriography, according to the method of previous reports, ${ }^{9,10}$ using an Agilent Technologies SONOS 5500 digital ultrasound system (Palo Alto, Calif) with a frequency of 5 to $12 \mathrm{MHz}$ (S12 ultraband transducer; Doppler frequency: $6 \mathrm{MHz}$ ). In color Doppler flow mapping, velocity range was set at $\pm 14.0 \mathrm{~cm} / \mathrm{s}$. The color gain was adjusted to provide optimal images. The acoustic window was located around the midclavicular line in the fourth or fifth intercostal spaces in the left lateral decubitus position. Anastomoses between the ITA and LAD were observed by two methods, one that was directed toward the anastomosis site from the distal LAD and another that was directed toward the anastomosis site from the stem of the ITA. In the first method, the ultrasound beam was transmitted toward the heart to visualize coronary blood flow in the LAD by color Doppler echocardiography. First, the left ventricle was imaged in the long-axis cross section and then the ultrasound beam was inclined laterally. Next, coronary blood flow in the distal portion of the LAD was searched under the guidance of color Doppler flow mapping. In the second method, long-axis images of the left ventricle were obtained, and then the anterior area to the right ventricle outflow tract and the anterior interventricular sulcus were carefully examined using combined imaging and color flow mapping. The ITA graft was identified as a tubular structure with color flow directed from the base to the apex and contained characteristic Doppler flow signals. Once the position of the distal portion of the LAD or ITA was identified, the anastomosis between the ITA and the LAD could be easily found.

We measured the diameters of the ITA and LAD using intraluminal flow signals obtained by a pulsed Doppler method, and we also measured flow velocity with a sample volume $(1.5$ or $2.0 \mathrm{~mm}$ wide) positioned on the color signals in the LAD, ITA, and anastomosis. Doppler spectral tracings of flow velocity in each were recorded by fast Fourier transformation analysis. The spectral Doppler display of the LAD flow showed a characteristic biphasic flow pattern, with a larger diastolic component and a smaller systolic one. The results were continuously recorded on half-inch super-VHS videotape for off-line analysis.

\section{Coronary Arteriography}

Coronary arteriography was performed by the Judkins technique after intravenous injection of $3000 \mathrm{IU}$ of heparin. The anastomosis was evaluated by use of multiple projections by an experienced investigator unaware of the echocardiographic data. A stenosis in the anastomotic site was considered significant if there was more than $25 \%$ narrowing of lumen diameter in at least 1 projection.

\section{Statistical Analysis}

Data are expressed as mean and standard deviation. Differences between the 2 groups were tested using the paired Student $t$ test.

\section{Results}

Under the guidance of color Doppler flow mapping, adequate spectral Doppler recordings of coronary flow in anastomoses between the ITA and LAD were obtained in 31 (89\%) of 35 study patients. As for coronary arteriography findings in those 31 patients, 26 (81\%) had no abnormal findings around the anastomosis. Further, 4 patients had a stringed ITA without anastomotic stenosis and 2 had $50 \%$ stenosis in the anastomotic sites.

In normal anastomosis patients, we were able to detect the ITA, LAD, and anastomotic site, as well as make accurate measurements of blood flow velocity (Figures 1, 2, 3 , and 4). The diameter of the ITA was $1.5 \pm 0.3 \mathrm{~mm}$ and that of the LAD was $2.0 \pm 0.4 \mathrm{~mm}$. Peak flow velocity in 

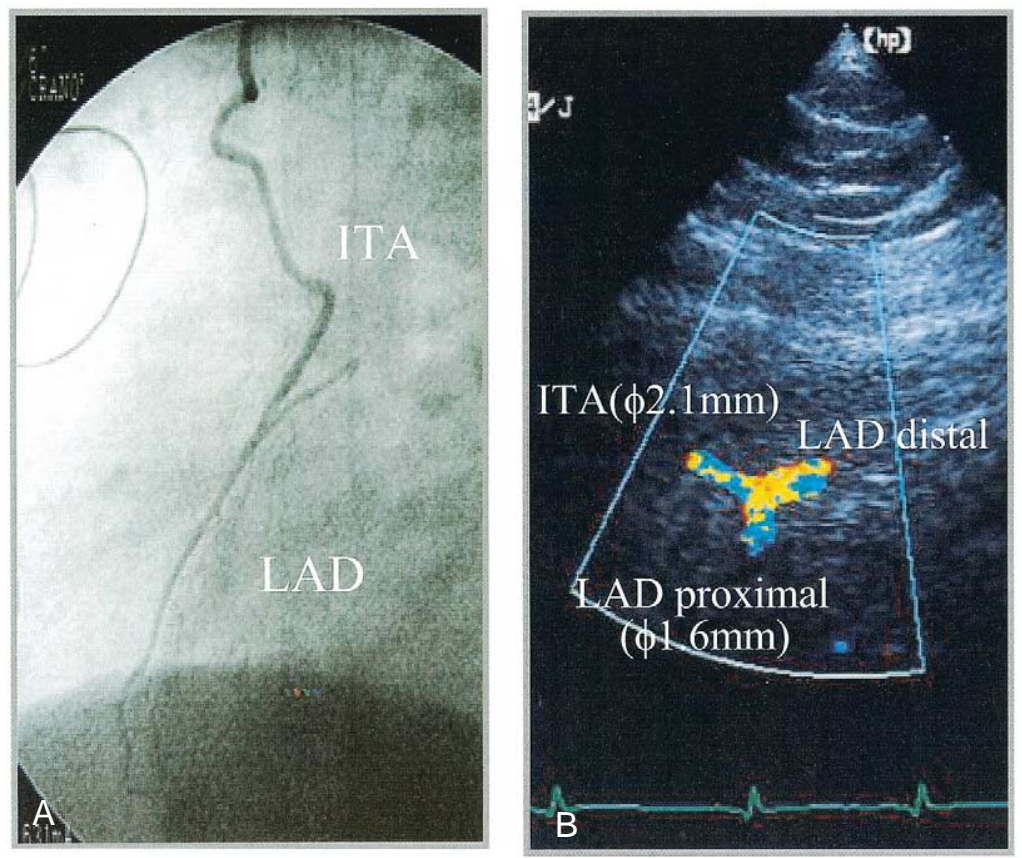

Figure 1. A typical case with a normal anastomosis between the internal thoracic artery (ITA) and left anterior descending coronary artery (LAD). A coronary arteriogram shows good anastomosis (A). The anastomotic site can be clearly seen by echocardiography (B).

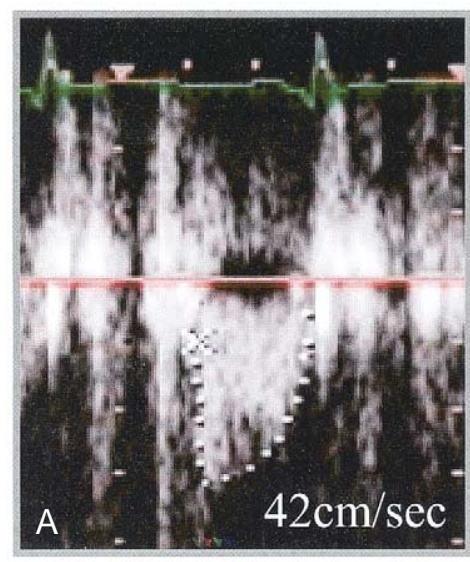

ITA anastomotic site

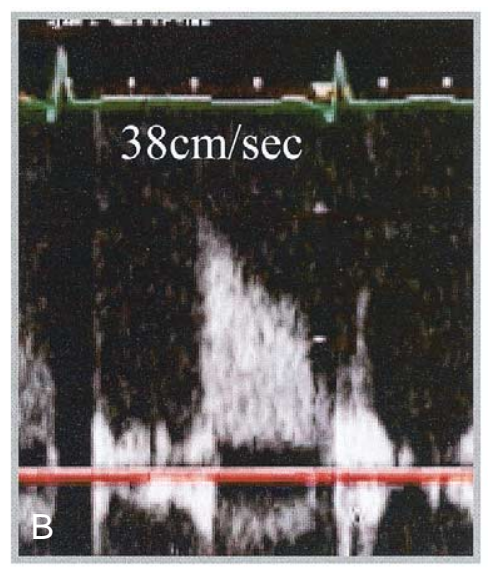

LAD distal

Figure 2. Flow velocity in the anastomotic site $(A)$ and the distal site of the left anterior descending coronary artery (LAD) (B) were nearly equal values. ITA, Internal thoracic artery.

the ITA was $58 \pm 25 \mathrm{~cm} / \mathrm{s}$ and in the LAD at the distal portion of the anastomotic site it was $47 \pm 20 \mathrm{~cm} / \mathrm{s}$; in the anastomotic site it was $59 \pm 29 \mathrm{~cm} / \mathrm{s}$.

Stringed ITAs were found in 4 patients because of good native LAD flow. In their angiographic findings, the ITA was very thin and blood flow from the ITA graft did not fill the LAD against the good native LAD flow. As a result, the LAD was shown to be very thin. In contrast, echocardiographic findings revealed that the LAD had a good blood flow, although the ITA was very thin (Figures 5 and 6). 

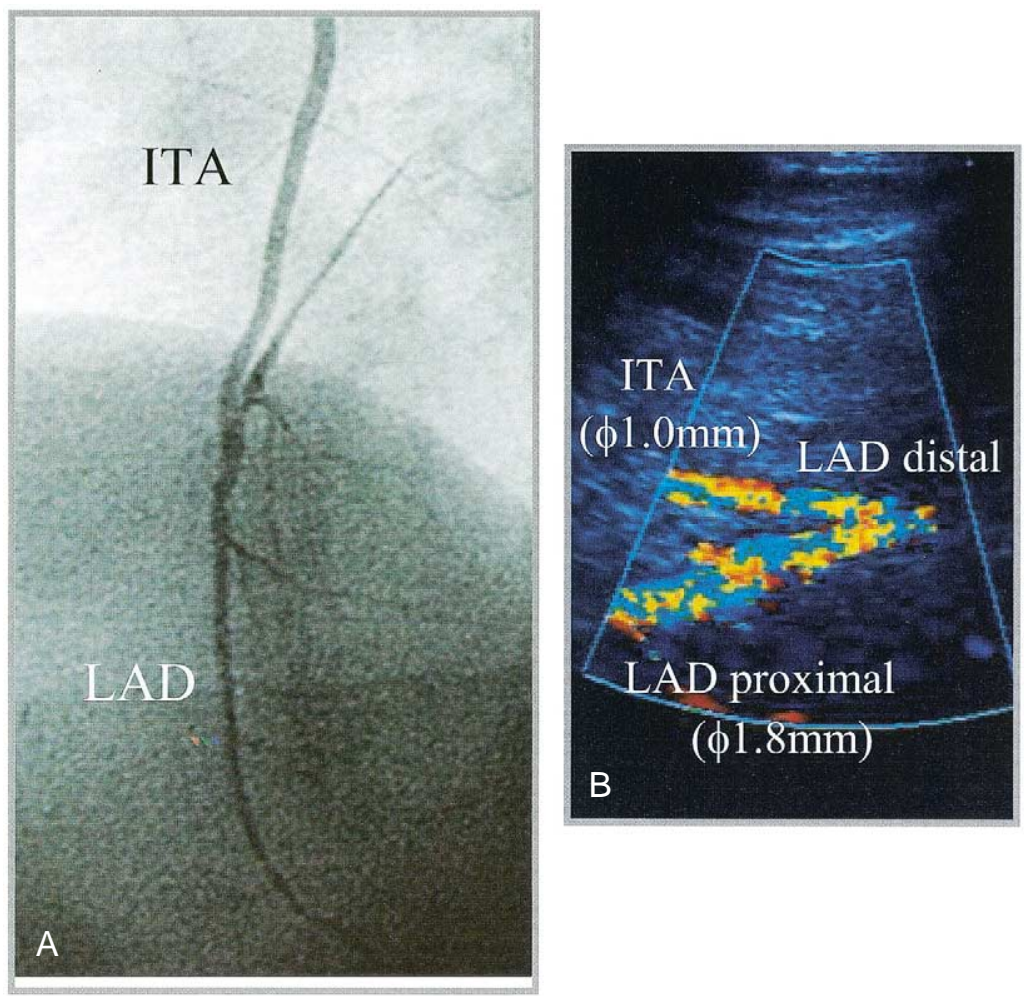

Figure 3. Another typical case of normal anastomosis between the internal thoracic artery (ITA) and left anterior descending coronary artery (LAD). A coronary arteriogram shows good anastomosis (A). The anastomotic site can be clearly seen by echocardiography (B).

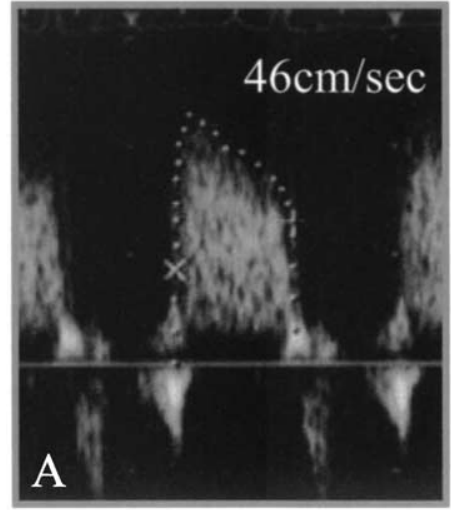

ITA

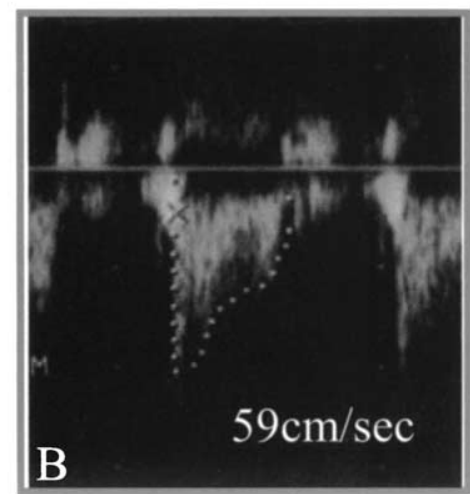

anastomotic site

Figure 4. Flow velocity in the internal thoracic artery (ITA) (A) and the anastomotic site (B) were nearly equal values. $L A D$, Left anterior descending coronary artery.

A stenotic anastomosis was found in 2 patients and blood flow velocity findings at these anastomotic sites $(83 \pm 228$ $\mathrm{cm} / \mathrm{s})$ was higher than in normal patients $(59 \pm 29 \mathrm{~cm} / \mathrm{s}$; Figures 7 and 8).

Interobserver and intraobserver variability for the detection of the anastomoses and measurements of Doppler velocity recordings were $2.8 \%$ and $2.0 \%$, respectively.

\section{Discussion}

The results of the present study demonstrate that transthoracic Doppler echocardiography is able to detect the ITA, $\mathrm{LAD}$, and anastomotic site, as we obtained a description in 31 (89\%) of 35 patients. Thus, echocardiographic findings were considered to be reliable after comparing with those of coronary arteriography. 

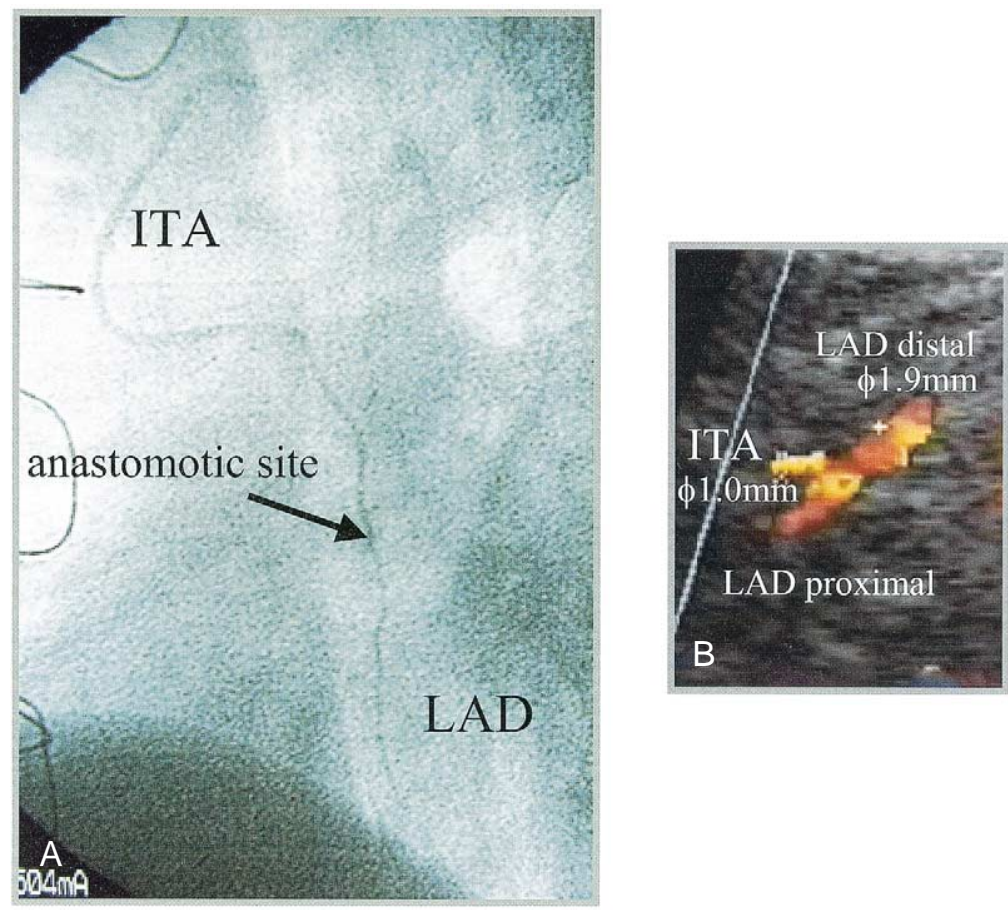

Figure 5. A case of stringed internal thoracic artery (ITA). A coronary arteriogram shows a stringed ITA and a very thin left anterior descending coronary artery $(L A D)(A)$. However, the echocardiographic study revealed a thin ITA and the LAD with good blood flow (B).

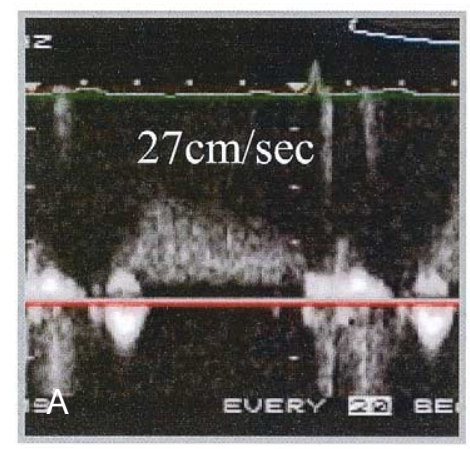

ITA

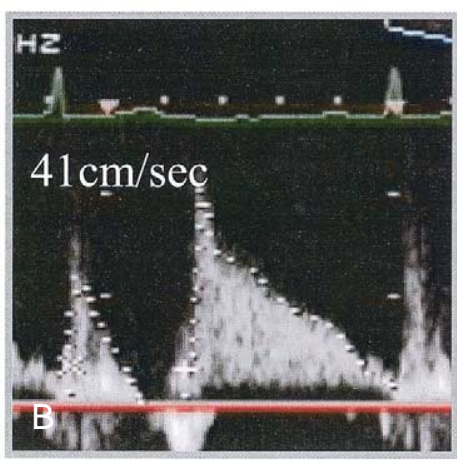

anastomotic site

Figure 6. A case of stringed internal thoracic artery (ITA). Flow velocity in the ITA (A) was slightly slower, but that in the anastomotic site (B) was within normal range. $L A D$, Left anterior descending coronary artery.

\section{Comparison with Coronary Arteriography}

This echocardiographic study provided a greater amount of information regarding the physiologic states in the area of anastomosis in patients at bedsides. Blood flow comes to the anastomotic site from both the stenosed native LAD and ITA, and those 2 compete with each other. In angiographic study, contrast medium must be injected with a certain amount of force, and thus an accurate analysis of this competition cannot be made (for example, regarding the blood flow distribution of the proximal and distal sides of the anastomosis). However, in our patients with stringed ITAs (found in 3 patients who underwent CABG using a traditional cardiopulmonary bypass and in 1 patient who underwent CABG without cardiopulmonary bypass), echocardiographic findings revealed a competitive relationship between the ITA flow and LAD flow in a more 

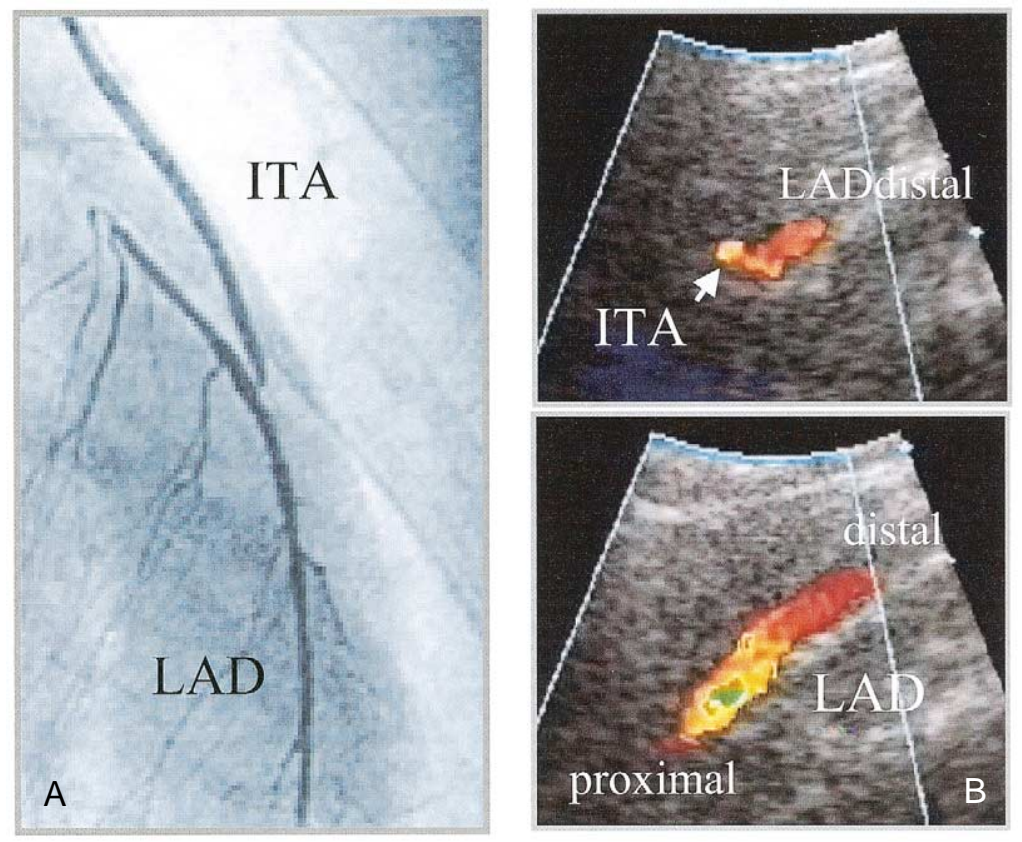

Figure 7. A case of stenosed anastomosis. A coronary arteriogram showing $50 \%$ stenosed anastomosis (A). The echocardiographic study shows a thin, internal thoracic artery (ITA) (B). LAD, Left anterior descending coronary artery.

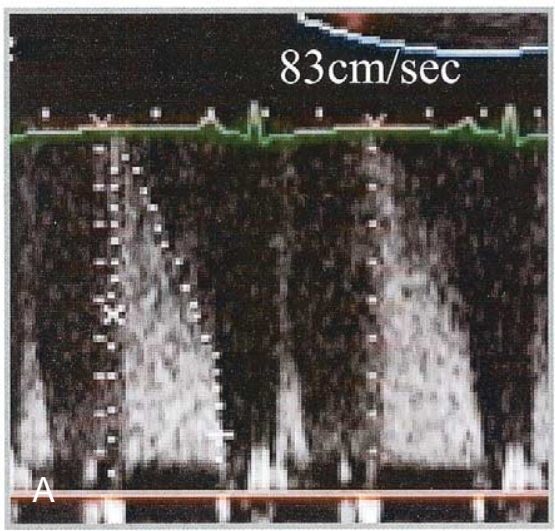

Anastomotic site

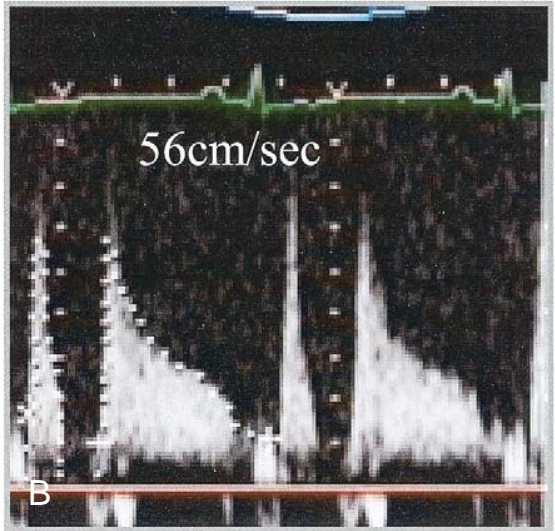

Distal site

Figure 8. A case of stenosed anastomosis. Flow velocity at the anastomotic site (A) was significantly faster, but that in the distal site of left anterior descending coronary artery (LAD) (B) was within normal range. ITA, Internal thoracic artery.

physiologic manner. Stringed ITAs were found in 3 patients with $75 \%$ stenosis in the left main trunk and in 1 patient with the regression of the stenosis in the LAD after surgery.

In addition, echocardiographic study provides better quantitative analysis of stenosis at anastomotic sites because it allows for a flow velocity measurement of the anastomotic sites. If stenosis exists at the anastomosis, a high flow velocity will be seen, as demonstrated in 2 patients with stenotic anastomosis in the present study, whose flow velocity rates were higher than in the normal anastomosis patients.

Several studies have reported that epicardial blood flow velocity can be measured by transthoracic Doppler echocardiography, which is widely used in clinical settings. ${ }^{4-8}$ To the best of our knowledge, the present study is the first to 
report investigation of an anastomosis between the ITA and LAD by this noninvasive method.

\section{Study Limitation}

In a certain number of cases, the $\theta$ angle was quite large $\left(>30^{\circ}\right)$, causing underestimation of the true velocity. In general, $\theta$ angle less than $30^{\circ}$ keeps the percent error within $14 \%$, based on simplified Bernoulli equation. However, the technologies of Agilent Technologies enables angle correction by the ultrasound system to downshift the peak velocity overestimation, and as a result, the percent error was under $10 \%$ for $\theta$ angle $<50^{\circ} .{ }^{11}$ A $\theta$ angle close to $0^{\circ}$ is very important; however, we believe that this method can be applied clinically with a high degree of accuracy. Furthermore, in the present study, there were only a small number of patients with significant stenosis in the anastomosis and a small number with stringed ITAs. In a future investigation, a greater number of patients should be studied by the present method to clarify our results.

We used 2-dimensional echocardiography and analyzed only 1 sectional plane. In coronary arteriography, the anastomosis was evaluated by use of multiple projections by an experienced investigator unaware of the echocardiographic data. However, we could analyze flow velocity and obtain a better quantitative assessment of the degree of stenosis at the site of anastomosis. Therefore, we believe that our method corresponds to coronary arteriography although it is important to note that we could detect the anastomosis between the ITA and LAD in only $89 \%$ of our patients, which was significantly different from coronary arteriography.

\section{Clinical Implications}

This totally noninvasive method has great potential for a variety of clinical applications and may provide additional information for cardiac catheterization at the bedside or in outpatient clinics. It could also be available for use during follow-up in an outpatient setting, and the use of intravenous ultrasonic contrast agents would allow for a detailed assessment of anastomoses.

\section{Conclusion}

The present study demonstrated that transthoracic Doppler echocardiography allowed the detection of the ITA, LAD, and anastomotic sites, as well as an accurate measurement of blood flow velocity in over $80 \%$ of our patients. This totally noninvasive method compares favorably with coronary arteriography and is considered to be reliable and able to provide a greater amount of information regarding the physiologic state in the area of anastomosis, such as a competitive relationship.

\section{References}

1. Lytle BW, Loop FD, Cosgrove DM, Ratliff NB, Easley K, Taylor PC. Long-term ( 5 to 12 years) serial studies of internal mammary artery and saphenous vein coronary bypass grafts. J Thorac Cardiovasc Surg. 1985;89:248-58.

2. Loop FD, Lytle BW, Cosgrove DM, et al. Influence of the internal mammary artery graft on 10 year survival and other cardiac events. N Engl J Med. 1986;314:1-6.

3. Acinapura AJ, Jacobowitz IJ, Kramer MD, Zisbrod Z, Cunningham JN. Internal mammary artery bypass: thirteen years of experience. Influence of angina and survival in 5,125 patients. J Cardiovasc Surg (Torino). 1992;33:554-9.

4. Fusejima K, Takahara Y, Sudo Y, Murayama H, Masuda Y, Inagaki Y. Comparison of coronary hemodynamics in patients with internal mammary artery and saphenous vein coronary artery bypass grafts: a noninvasive approach using combined two-dimensional and Doppler echocardiography. J Am Coll Cardiol. 1990;15:131-9.

5. Takagi T, Yoshikawa J, Yoshida K, Akasaka T. Noninvasive assessment of left internal mammary artery graft patency using duplex Doppler echocardiography from supraclavicular fossa. J Am Coll Cardiol. 1993;22:1647-52.

6. Ross JJ Jr, Mints RG, Chandrasekaran K. Transthoracic two-dimensional high frequency $(7.5 \mathrm{MHz})$ ultrasonic visualization of the distal left anterior coronary artery. J Am Coll Cardiol. 1990;15:373-7.

7. Kenny A, Shapiro LM. Transthoracic high frequency two-dimensional echocardiography, Doppler and color flow mapping to determine anatomy and blood flow patterns in the distal left anterior descending coronary artery. Am J Cardiol. 1992;69:1265-8.

8. Kenny A, Wisbey CR, Shapiro LM. Measurement of left anterior descending coronary artery flow velocities by transthoracic Doppler ultrasound. Am J Cardiol. 1994;73:12021-2.

9. Hozumi T, Yoshida K, Ogata Y, et al. Noninvasive assessment of significant left anterior descending coronary artery stenosis by coronary flow velocity reserve with transthoracic color Doppler echocardiography. Circulation. 1998;97:1557-62.

10. Hozumi T, Yoshida K, Akasaka T, et al. Noninvasive assessment of coronary flow velocity and coronary flow velocity reserve in the left anterior descending coronary artery by Doppler echocardiography: comparison with invasive technique. J Am Coll Cardiol. 1998;32: 1251-9.

11. Daigle RJ, Stavros T, Lee RM. Overestimation of velocity and frequency values by multielement linear array Dopplers. J Vasc Technol. 1990;14:206-13 